\title{
QUASI-ALMOST CONVERGENCE IN A NORMED SPACE
}

\author{
Dimitrije Hajduković
}

\begin{abstract}
In [1] was shown the existence of the functionals of the kind of Banach limits defined on the real vector space $\mathbf{m}$ of all bounded sequences in a real normed space $X$. In [2] by these functionals was defined the almost convergence of a sequence $\left(x_{i}\right) \in \mathbf{m}$ and shown that $\left(x_{i}\right)$ almost converges to $s \in X$ iff

$$
\left\|\frac{1}{p} \sum_{i=0}^{p-1} X_{k+i}-s\right\|_{X} \rightarrow 0 \quad \text { as } \quad p \rightarrow \infty
$$

uniformly in $k(=0,1,2, \ldots)$.

2. The basic idea in this paper is to obtain a new method of summability of the vector sequences $\left(x_{i}\right) \in \mathbf{m}$. The paper is organized as follows. First, we will show the existence of an another family of functionals (of the kind of Banach limits) defined on the space $\mathbf{m}$. Next, we define, by these functionals, a new method of summability of sequences $\left(x_{i}\right) \in \mathbf{m}$ which will be called quasi almost convergence. Further, we will show a theorem which contains a necessary and sufficient condition for a sequence $\left(x_{i}\right) \in$ m to be quasi almost convergent. Next, we shall prove two theorems which shows that the class of quasi almost convergent sequences lies between the class of almost convergent sequences and the class of Csummable sequences.
\end{abstract}

\section{A family of functionals of the kind of Banach limits}

Let us define on the space $\mathbf{m}$ the functional $q$ by

$$
\left.q(x) \equiv q\left(x_{i}\right)=\varlimsup_{p \rightarrow \infty}\left\{\sup _{n} \frac{1}{p}\left\|\sum_{i=n p}^{(n+1) p-1} x_{i}\right\|_{X}\right\} \quad\left(x_{i}\right)=x \in \mathbf{m}\right),
$$

Key words and phrases: Functional, Banach limits, $C$-summable sequences. 
where the supremum is taken over all $n(=0,1,2, \ldots)$. The functional $q$ clearly is real-valued and it satisfies the conditions

$$
q(x) \geq 0, \quad q(a x)=|a| q(x), \quad q(x+y) \leq q(x)+q(y) \quad(a \in R ; \quad x, y \in \mathbf{m}) ;
$$

that is, $q$ is a symmetric convex functional on the space $\mathbf{m}$. According to a corollary of Hahn-Banach theorem (see also [3], Esercise 11.2, p.187) must exist a nontrivial linear functional $L$ on the space $\mathbf{m}$ such that

$$
\left|L\left(x_{i}\right)\right| \leq q\left(x_{i}\right) \quad\left(\left(x_{i}\right) \in \mathbf{m}\right) .
$$

Next, let us define the sequence $y=\left(y_{i}\right)$ by

$$
y_{i}=x_{i+1}-x_{i} \quad(i=0,1,2, \ldots) .
$$

Then, by (1), we have

$$
q\left(y_{i}\right)=\varlimsup_{p \rightarrow \infty}\left\{\sup _{n} \frac{1}{p}\left\|x_{(n+1) p}-x_{n p}\right\|\right\} \leq \lim _{p \rightarrow \infty} \frac{2}{p} \sup _{i}\left\|x_{i}\right\|_{X}=0 .
$$

Whence, by (2), we obtain

$L\left(y_{i}\right)=L\left(x_{i+1}-x_{i}\right)=L\left(x_{i+1}\right)-L\left(x_{i}\right)=0 \quad$ or $\quad L\left(x_{i+1}\right)=L\left(x_{i}\right), \quad\left(x_{i}\right) \in \mathbf{m}$.

Further, we wish to show that the functional $L$, satisfying the above conditions, is not unique. To do this denote by $\mathbf{m}_{0}$ the subspace of all sequences $\left(x_{i}\right) \in \mathbf{m}$ having $\lim _{i \rightarrow \infty} x_{i}=0 \in X$. Then clearly we have

$$
q\left(x_{i}\right)=L\left(x_{i}\right)=0 \quad,\left(x_{i}\right) \in \mathbf{m}_{0} .
$$

Next, let us choose a point $s \in X(s \neq 0)$ and define the sequence $y=\left(y_{i}\right)$ by $y_{i}=s(i=0,1,2, \ldots)$. Then $y \in \mathbf{m B S} \mathbf{m}_{0}$ and $q(y)=\|s\|>0$. To extend the functional $L: \mathbf{m}_{0} \rightarrow R$ to the space spanned by $\mathbf{m}_{0}$ and $\{y\}$ (that is, the space $\mathbf{m}_{0} \cup\{y\}$ ), the value $L\left(y_{i}\right)$ we can choose arbitrarily in the segment $\left[-q\left(y_{i}\right), q\left(y_{i}\right)\right]$. Thus, the functional $L$ can be extended such that it has distinct values at the point $y \in \mathbf{m}$. This means that there are distinct functionals on $\mathbf{m}$ satisfying the above conditions. Indeed, we can take the value $L(y)$ arbitrarily in the segment $[k, K]$, where

$$
k=\sup _{x \in m_{0}}\{-q(x+y)\}, \quad K=\inf _{x \in m_{0}}\{q(x+y)\}
$$

since $L(x)=0, \forall x \in \mathbf{m}_{0}$ (see, for example, [4], p.222). Further, by (1) we have $q(x+y)=q(y)=q\left(y_{n}\right)$ since the sequence $x+y=\left(x_{i}\right)+y$ converges to $s$. Consequently, we can take arbitrarily $L(y)$ in $\left[-q\left(y_{i}\right), q\left(y_{i}\right)\right]$.

The following lemma is well known in the literature.

Lemma. Let $X$ be a real linear space and $q: X \rightarrow R$ a functional such that the following assertions are valid

$$
q(x) \geq 0, \quad q(a x)=|a| q(x), \quad q(x+y) \leq q(x)+q(y) \quad(a \in R ; \quad x, y \in X) .
$$


Then, for each $x_{0} \in X$, there exists a linear functional $L$ on $X$ such that

$$
(\forall x \in X)|L(x)| \leq q(x), \quad L\left(x_{0}\right)=q\left(x_{0}\right) .
$$

Denoting now by $\Pi$ the family of functionals satisfying the above conditions, then for each $s \in X$ we have

$$
(\forall L \in \Pi) \quad L\left(x_{i}-s\right)=0 \quad \text { iff } \quad q\left(x_{i}-s\right)=0 \quad\left(\left(x_{i}\right) \in \mathbf{m}\right) .
$$

Indeed, $q\left(x_{i}-s\right)=0$ clearly implies $L\left(x_{i}-s\right)=0, \forall L \in \Pi$. Also, the implication

$$
(\forall L \in \Pi) \quad L\left(x_{i}-s\right)=0 \Rightarrow q\left(x_{i}-s\right)=0
$$

is equivalent to the implication

$$
q\left(x_{i}-s\right)>0 \Rightarrow(\exists L \in \Pi) \quad L\left(x_{i}-s\right) \neq 0
$$

which, by the lemma proved before, is valid. So, (4) is true.

The results obtained before we summarize by the following statement.

Theorem 1. There exists the family $\Pi$ of nontrivial functionals $L$ defined on the space $\mathbf{m}$ such that for ali $a, b \in R$, each $s \in X$ and all $\left(x_{n}\right),\left(y_{n}\right) \in$ $\mathbf{m}$, the following assertions are valid

$$
\begin{aligned}
& 1^{\circ} L\left(a x_{i}+b y_{i}\right)=a L\left(x_{i}\right)+b L\left(y_{i}\right), \\
& 2^{\circ} L\left(x_{i+1}\right)=L\left(x_{i}\right), \\
& 3^{\circ}\left|L\left(x_{n}\right)\right| \leq q\left(x_{n}\right), \\
& 4^{\circ}(\forall L \in \Pi) L\left(x_{i}-s\right)=0 \text { iff } q\left(x_{i}-s\right)=0 .
\end{aligned}
$$

Having obtained the functionals $L \in \Pi$ we can proceed to the investigation of the sequences $\left(x_{i}\right) \in \mathbf{m}$ which ail the functionals $L \in \Pi$ assigned same value.

\section{Quasi-almost convergence}

The following definition gives a natural extension of aimost convergence of a sequence $\left(x_{i}\right) \in \mathbf{m}$.

Definition 1. A sequence $\left(x_{i}\right) \in \mathbf{m}$ is quasi almost convergent to $s \in$ $X$ or quasi $F$-summable to $s$ and $s$ is its quasi aimost limit (we will write $\left.(Q-F)-\lim _{i \rightarrow \infty} x_{i}=s\right)$ if

$$
(\forall L \in \Pi) \quad L\left(x_{i}-s\right)=0 .
$$

Let us show that quasi aimost limit of a sequence defined in such way is unique. To do this, suppose $s^{\prime}$ and $s$ " are any two quasi aimost limits of a sequence $\left(x_{i}\right) \in \mathbf{m}$ and define the sequences $\left(y_{i}\right)$ and $\left(z_{i}\right)$ by

$$
\begin{aligned}
& y_{i}=s^{\prime} \\
& z_{i}=s^{\prime \prime}
\end{aligned} \quad i=1,2, \ldots
$$


Then, by (5),

$(\forall L \in \Pi) \quad L\left(z_{i}-y_{i}\right)=L\left(x_{i}-y_{i}\right)-L\left(x_{i}-z_{i}\right)=L\left(x_{i}-s^{\prime}\right)-L\left(x_{i}-s^{\prime \prime}\right)=0$ which, by (4) and (1), implies

$$
q\left(z_{i}-y_{i}\right)=\left\|s^{\prime}-s^{\prime \prime}\right\|=0 \text { or } s^{\prime}=s^{\prime \prime} .
$$

Example. Let $0 \in X$ be the aditive identity element of the vector adition. Next, let $s \in X(s \neq 0)$ and fix it. Then the sequence

$$
\left(x_{i}\right): s, 0, s, 0, \ldots
$$

converge quasi-almost and $(Q-F)-\lim _{i \rightarrow \infty} x_{i}=\frac{1}{2} s$.

Indeed, by the theorem 1 we have

$$
(\forall L \in \Pi) L\left(x_{i+1}\right)=L\left(x_{i}\right)
$$

thus is

$$
(\forall L \in \Pi) L\left(x_{i}\right)=\frac{1}{2} L\left(x_{i}+x_{1+i}\right)=\frac{1}{2} L(x),
$$

and

$$
(\forall L \in \Pi) L\left(x_{i}-\frac{1}{2} s\right)=L\left(x_{i}\right)-\frac{1}{2} L(s)=0 .
$$

Hence, by the definition 1 , we have $(Q-F)-\lim _{i \rightarrow \infty} x_{i}=\frac{1}{2} s$.

Now, we will show the follovving theorem containing a necessary and sufficient condition for a sequence $\left(x_{i}\right) \in \mathbf{m}$ to be quasi almost convergent.

Theorem 2. A sequence $\left(x_{i}\right) \in \mathbf{m}$ quasi almost converges to $s \in X$ iff

$$
\left\|\frac{1}{p} \sum_{i=n p}^{(n+1) p-1} x_{i}-s\right\|_{X} \rightarrow 0 \quad \text { as } \quad p \rightarrow \infty
$$

uniformly in $n(=0,1,2, \ldots)$.

Proof. Suppose, for a sequence $\left(x_{i}\right) \in \mathbf{m}$, we have $(Q-F)-\lim _{i \rightarrow \infty} x_{i}=$ $s$. Then, by (5) and (4), we have $q\left(x_{i}-s\right)=0$ or, by (1),

$$
\varlimsup_{p \rightarrow \infty}\left\{\sup _{n} \frac{1}{p}\left\|\sum_{i=n p}^{(n+1) p-1}\left(x_{i}-s\right)\right\| \|_{X}\right\}=0 .
$$

Thence, for any $\varepsilon>0$ there exists an integer $p_{0}>0$ such that for all $p$ and $n\left(p>p_{0}, n=0, l, 2, \ldots\right)$, we have

$$
\frac{1}{p}\left\|\sum_{i=n p}^{(n+1) p-1}\left(x_{i}-s\right)\right\|_{X}<\varepsilon .
$$


Since $\varepsilon>0$ is arbitrary, we have

$$
\left\|\frac{1}{p} \sum_{i=n p}^{(n+1) p-1} x_{i}-s\right\|_{X} \rightarrow 0 \quad \text { as } \quad p \rightarrow \infty
$$

uniformly in $n$; so, the condition (6) is necessary. Conversely, let the condition (6) be true. This means that

or

$$
\sup _{n} \frac{1}{p}\left\|\sum_{i=n p}^{(n+1) p-1}\left(x_{i}-s\right)\right\|_{X} \rightarrow 0 \quad \text { as } \quad p \rightarrow \infty
$$

$$
q\left(x_{i}-s\right)=\lim _{p \rightarrow \infty}\left\{\sup _{n} \frac{1}{p}\left\|\sum_{i=n p}^{(n+1) p-1}\left(x_{i}-s\right)\right\|_{X}\right\}=0 .
$$

Hence, by (4), we have

$$
(\forall L \in \Pi) \quad L\left(x_{i}-s\right)=0,
$$

which, by (5), means that $(Q-F)-\lim _{i \rightarrow \infty} x_{i}=s$; so, the condition (6) is sufficient. The proof is now complete.

Remark 1. We here take, by definition, that a sequence $\left(x_{i}\right), x_{i} \in X$ $(i=0,1,2, \ldots)$ is $C$-summable to $s \in X$ iff

$$
\left\|\frac{1}{p} \sum_{i=n p}^{(n+1) p-1} x_{i}-s\right\|_{X} \rightarrow 0 \text { as } p \rightarrow \infty .
$$

Now, we show the following two theorems that show that the class of quasi almost convergent sequences lies between the class of almost convergent sequences and the class of $C$-summable sequences.

Theorem 3. If a sequence $\left(x_{i}\right) \in \mathbf{m}$ almost converges to $s \in X$, then it quasi almost converges to $s$.

Proof. Let a sequence $\left(x_{i}\right) \in \mathbf{m}$ almost converges to $s \in X$. Then, by (0.1), for any $\varepsilon>0$ there exists an integer $p_{0}>0$ such that

$$
\left\|\frac{1}{p} \sum_{i=0}^{p-1} x_{k+1}-s\right\|_{X}<\varepsilon \quad\left(p>p_{0}, \quad k=0,1,2, \ldots\right) .
$$

Hence for $k=n p\left(p>p_{0}, n=0,1,2 \ldots\right)$ we have

$$
\left\|\frac{1}{p} \sum_{i=0}^{p-1} x_{n p+i}-s\right\|_{X}=\left\|\frac{1}{p} \sum_{i=n p}^{(n+1) p-1} x_{i}-s\right\|_{X}<\varepsilon .
$$


Since $\varepsilon>0$ is arbitrary, we have

$$
\left\|\frac{1}{p} \sum_{i=n p}^{(n+1) p-1} x_{i}-s\right\|_{X} \rightarrow 0 \quad \text { as } \quad p \rightarrow \infty
$$

uniformly in $n$ which, by (6), means that $\left(x_{i}\right)$ quasi almost converges to $s$.

Theorem 4. If a sequence $\left(x_{i}\right) \in \mathbf{m}$ quasi almost converges to $s \in X$, then it is $C$-summable to $s$.

Proof. Let $\left(x_{i}\right) \in \mathbf{m}$ quasi almost converges to $s \in X$. Then (6) is true which for $n=0$ implies (7); so, $\left(x_{i}\right)$ is $C$-summable to $s$.

Remark 2. From the comparison of the definition of the almost-convergence and the definition 1 , follows that there is a very big possibility that there exist sequences that converge quasi-almost, but not almost. Proof of that is still an open problem.

Clearly, the similar remark also stands for relationship of the quasialmost convergence and the $C$-summability.

\section{References}

[1] Hajduković D.: The functionals of the kind of Banach limits, Publications de L'Institut Mathematique, T. 19 (33), 1975.

[2] Hajduković D.: Almost convergence of vector sequences, Математички весник 12 (27), 1975. 245-249.

[3] Bachman G. and Narici L.: Functional Analysis, Academic Press, New York and London (1966), p. 181.

[4] Aljančić S.: Uvod u realnu i funkcionalnu analizu, Gradevinska knjiga, Beograd, $1968,222-224$.

Milana Rakića 15

51000 Banja Luka

$\mathrm{BiH}$

Received Decembar 7, 2002. 